\title{
Robust MPC of constrained nonlinear systems based on
}

\section{interval arithmetic}

\author{
D. Limon ${ }^{\dagger}$, J.M. Bravo*, T. Alamo ${ }^{\dagger}$ and E.F. Camacho ${ }^{\dagger}$ \\ † Departamento de Ingeniería de Sistemas y Automática, Universidad de Sevilla \\ Escuela Superior de Ingenieros, Camino de los Descubrimientos s/n, 41092 Sevilla. Spain \\ Telephone: +34 954487347, Fax: +34954487340 \\ email: $\{$ limon, alamo, eduardo\}@cartuja.us.es \\ * Departamento de Ingeniería Electrónica, de Sistemas informáticos y Automática \\ Universidad de Huelva
}

Escuela Politécnica Superior de La Rábida. Ctra. Huelva-Palos de la Frontera, Huelva. Spain

Telephone: +34 959017367, email: caro@uhu.es

\begin{abstract}
A robust MPC for constrained discrete-time nonlinear systems with additive uncertainties is presented. The proposed controller is based on the concept of the reachable sets, that is, the sets that contain the predicted evolution of the uncertain system for all possible uncertainties. If processes are nonlinear these sets are very difficult to compute. A conservative approximation based on interval arithmetic is proposed for the on-line computation of these sets. This technique provides good results with a computational effort only slightly greater than the one corresponding to the nominal prediction.

These sets are incorporated in the MPC formulation in order to achieve robust stability. By choosing a robust positively invariant set as a terminal constraint, a robustly stabilizing controller is obtained. Stability is guaranteed in case of suboptimality of the computed solution. In order to illustrate the proposed controller, it is applied to a Continuous Stirred Tank Reactor (CSTR) with an exothermic reaction.
\end{abstract}




\section{Introduction}

The main reasons for the success of Model Predictive Control (MPC) is that it is one of the few techniques that is able to handle explicitly constraints and model uncertainties. Furthermore, underlying theoretic problems on linear MPC and on nonlinear MPC are well studied [1]. See [2, 3] for a survey on the process industry application issues and $[4,5]$ for a survey on nonlinear MPC. Particularly interesting is [1] where a standard formulation of the MPC is established and sufficient conditions to guarantee asymptotic stability are given.

Although it has been proved that the controller has some degree of robustness [6, 7], if the system differs from the prediction model, the stabilizing properties may be lost. In order to get robust stability when uncertainties are present, they must be taken into account in the computation of the control law. Two different approaches have been proposed: open-loop and closed-loop MPC.

In the open-loop MPC formulation the decision variables are a sequence of control actions as in the nominal case. Any feasible sequence applied in an open-loop manner must steer the system to the terminal region in an admissible way for any possible uncertainty. Then the reaction of the controller to the uncertainty (due to the feedback structure) is not considered in the predictions, which makes the controller quite conservative. Consequently, the domain of attraction may be small (or even empty) compared with the real robustly stabilizable region. In [8] an open-loop dual-mode MPC controller is proposed and robustness under decaying additive uncertainties is achieved.

This conservativeness can be overcome if a sequence of control laws is used as decision variables, which leads us to the closed-loop formulation. In this case, the problem is mitigated at expense of a quite more complex optimization problem to solve. The feasibility region is quite larger than the one of the open-loop formulation and it tends to the maximal robustly stabilizable region when the control horizon increases. In case of constrained linear systems, the closed loop MPC has been characterized [9] and explicit solutions of the controller can be obtained by means of multi-parametric programming (see [10] and references there in). In the case of nonlinear systems, the optimization problem is prohibitively complex and it must be considered as a merely theoretical controller.

In this paper, an open-loop robust MPC for constrained discrete-time nonlinear systems with additive bounded uncertainties is presented. It is based on the reachable sets: the sets which contain the predicted 
evolution of the uncertain system under any possible uncertainty. Since the nonlinearity of the model makes these sets difficult to be accurately obtained, conditions are established to compute them by using approximate procedures.

Interval arithmetic is used for the computation of the approximate reachable sets. This procedure is very useful for the on-line implementation of the proposed controller, since the computational effort is similar to the nominal prediction. Furthermore, quite good results are obtained since the method provides local approximations to the reachable set.

Based on these sets, a robustly stabilizing dual-mode MPC controller is proposed. The controller is based on the addition of a robust invariant set as a terminal constraint with an associated robust local control law. Thus, the dual-mode controller applies the MPC solution as control input when the state is not in the terminal region, and once the system has reached it, the local control law is applied. For all initial states such that the optimization problem is feasible, robust stability is guaranteed. Hence, the uncertain closed-loop system reaches the terminal region in a finite number of steps and it remains in it all the time. Robust stability is ensured in case of suboptimality of the solution.

The paper is organized as follows: In section 2, some preliminary results are established, the exact and approximate reachable sets are presented and some basic results in interval arithmetic are given; in section 3, the Robust MPC strategy is demonstrated, and in the following section, closed loop stability is proved. The application of the proposed controller to a CSTR is shown in section 5, and finally some conclusions are given at the end of the paper.

\section{Preliminary results}

\subsection{System description}

Consider an uncertain nonlinear discrete-time system given by

$$
\mathbf{x}_{k+1}=f\left(\mathbf{x}_{k}, \mathbf{u}_{k}\right)+\mathbf{w}_{k}
$$

where $\mathbf{x}_{k} \in \mathbb{R}^{n}$ is the state of the system and $\mathbf{u}_{k} \in \mathbb{R}^{m}$ is the control vector at sample time $k$. The vector $\mathbf{w}_{k} \in \mathbb{R}^{n}$ is the disturbance or uncertainty which is assumed to be additive and bounded in a compact set $W$ 
that contains the origin.

$$
\mathbf{w}_{k} \in W
$$

The system is subject to constraints on the state $\mathbf{x}_{k} \in X$ and on the control action $\mathbf{u}_{k} \in U$.

Note that the additive uncertainty can model perturbed systems and a wide class of model mismatches taking into account that these ones might depend on the state of the system, since

$$
\mathbf{x}_{k+1}=\tilde{f}\left(\mathbf{x}_{k}, \mathbf{u}_{k}\right)=f\left(\mathbf{x}_{k}, \mathbf{u}_{k}\right)+\Delta f\left(\mathbf{x}_{k}, \mathbf{u}_{k}\right) \Rightarrow \mathbf{w}_{k}=\Delta f\left(\mathbf{x}_{k}, \mathbf{u}_{k}\right) \in W, \quad \forall \mathbf{x}_{k} \in X, \mathbf{u}_{k} \in U
$$

where $X$ is a closed set and $U$ a compact set, both of them containing the origin.

The model given by

$$
\hat{\mathbf{x}}_{k+1}=f\left(\hat{\mathbf{x}}_{k}, \mathbf{u}_{k}\right)
$$

denotes the nominal model of the system. The vector $\mathbf{u}_{F}(k)$ denotes a sequence of control of $M$ inputs

$$
\mathbf{u}_{F}(k)=\{\mathbf{u}(k \mid k), \mathbf{u}(k+1 \mid k), \cdots, \mathbf{u}(k+M-1 \mid k)\}
$$

where the number of future inputs $M$ is derived from the context. For a given state $\mathbf{x}_{k}$ and a sequence of control actions $\mathbf{u}_{F}(k)$, the future state of the system at time $k+j$ predicted by using the nominal model is denoted as $\hat{\mathbf{x}}(k+j \mid k)$. Hence, $\hat{\mathbf{x}}(k+j+1 \mid k)=f(\hat{\mathbf{x}}(k+j \mid k), \mathbf{u}(k+j \mid k))$, where $\hat{\mathbf{x}}(k \mid k)=\mathbf{x}_{k}$.

\subsection{Reachable sets}

Since there are mismatches between the real system and the nominal model, the predicted evolution using the nominal model differs from the real evolution of the system. In order to consider this effect in the controller synthesis, it is interesting to compute the region around the nominal prediction that confines the state of the system under any possible uncertainties.

This idea is the basis of the so-called reachable sets. Consider that the state of the system at sample time $k$ is $\mathbf{x}_{k}$ and a sequence of control inputs $\mathbf{u}_{F}(k)$ is applied to the uncertain system. The evolution of the system depends on the uncertainties, that are known to belong to the bounded set $W$. The reachable set at sample time $k+j$ is denoted as $\mathcal{X}_{j}\left(\mathbf{x}_{k}, \mathbf{u}_{F}(k)\right)$. This set is the region that confines the evolution of the uncertain system under any possible realization of the uncertainties until sample time $k+j$, that is $\forall \mathbf{w}_{k+i} \in W$, for $i=0, \cdots, k+j-1$. Note that this set depends on $\mathbf{x}_{k}$, on the sequence of inputs from $k$ to $k+j-1$, i.e. $\{\mathbf{u}(k \mid k), \cdots, \mathbf{u}(k+j-1 \mid k)\}$ and on the set of uncertainties $W$. 
Hereafter some definitions and results related to the reachable sets are presented. First some notations are introduced; consider sets $A$ and $B \subset \mathbb{R}^{n}$, a vector $\mathbf{x} \in \mathbb{R}^{n}$ and a function $g(\mathbf{x}): \mathbb{R}^{n} \rightarrow \mathbb{R}^{n}$ then the following sets are defined: $\mathbf{x}+A=\{\mathbf{x}+\mathbf{a}, \mathbf{a} \in A\}, g(A)=\{g(\mathbf{a}), \mathbf{a} \in A\}, A+B=\{\mathbf{a}+\mathbf{b}, \mathbf{a} \in A, \mathbf{b} \in B\}$, and $A \sim B=\left\{\mathbf{c} \in \mathbb{R}^{n}: \mathbf{c}+B \subseteq A\right\}$.

Definition 1 (Reachable set) Consider a system (1) and consider a given state at sample time $k, \mathbf{x}_{k}$, and a sequence of control inputs $\mathbf{u}_{F}(k)$. Then the reachable set at sample time $k+j, x_{j}\left(\mathbf{x}_{k}, \mathbf{u}_{F}(k)\right)$, is given by the following recursion

$$
X_{j}\left(\mathbf{x}_{k}, \mathbf{u}_{F}(k)\right)=f\left(X_{j-1}\left(\mathbf{x}_{k}, \mathbf{u}_{F}(k)\right), \mathbf{u}(k+j-1 \mid k)\right)+W
$$

where $X_{1}\left(\mathbf{x}_{k}, \mathbf{u}_{F}(k)\right)=f\left(\mathbf{x}_{k}, \mathbf{u}(k \mid k)\right)+W$.

Note that $X_{j}\left(\mathbf{x}_{k}, \mathbf{u}_{F}(k)\right)$ is the set that contains the uncertain evolution of all the states of $X_{j-1}\left(\mathbf{x}_{k}, \mathbf{u}_{F}(k)\right)$, that is

$$
X_{j}\left(\mathbf{x}_{k}, \mathbf{u}_{F}(k)\right)=\bigcup_{\mathbf{x} \in X_{j-1}} f(\mathbf{x}, \mathbf{u}(k+j-1 \mid k))+W
$$

Due to the nonlinear nature of the model, for a given set $A \subseteq \mathbb{R}^{n}$ and a given control action $\mathbf{u}$, the set $f(A, u)$ is very difficult to compute and thus, the reachable sets are not useful from a practical point of view. In order to reduce the complexity of the computation, these sets can be substituted by tractable approaches denoted as approximate reachable sets. The approximation is based on a procedure $\psi(A, \mathbf{u})$ to compute a conservative and tractable approximation of $f(A, \mathbf{u})$ with a lower computational burden. This procedure must satisfy the following conditions.

Assumption 1 The approximate procedure $\psi(A, \mathbf{u})$, where $A \subseteq X$ and $\mathbf{u} \in U$ satisfies the following conditions:

- Inclusion condition: $f(A, \mathbf{u}) \subseteq \psi(A, \mathbf{u})$.

- Monotonic condition: If $B$ is a set such that $B \subseteq A$, then $\psi(B, \mathbf{u}) \subseteq \psi(A, \mathbf{u})$.

Based on this procedure, it is possible to compute conservative approximations to the reachable sets.

Definition 2 (Approximate reachable set) Consider a system (1) and a procedure $\psi(\cdot, \cdot)$ that satisfies assumption 1 for the system. Then for a given state at sample time $k, \mathbf{x}_{k}$, and a sequence of control inputs 
$\mathbf{u}_{F}(k)$, the approximate reachable set at sample time $k+j, \hat{x}_{j}\left(\mathbf{x}_{k}, \mathbf{u}_{F}(k)\right)$, is given by the following recursion

$$
\hat{X}_{j}\left(\mathbf{x}_{k}, \mathbf{u}_{F}(k)\right)=\psi\left(\hat{X}_{j-1}\left(\mathbf{x}_{k}, \mathbf{u}_{F}(k)\right), \mathbf{u}(k+j-1 \mid k)\right)+W
$$

where $\hat{X}_{1}\left(\mathbf{x}_{k}, \mathbf{u}_{F}\right)=f\left(\mathbf{x}_{k}, \mathbf{u}(k \mid k)\right)+W=X_{1}\left(\mathbf{x}_{k}, \mathbf{u}_{F}(k)\right)$.

These approximate reachable sets have the following properties.

Property 1 Consider a given state $\mathbf{x}_{k}$ and a sequence of $M$ control inputs $\mathbf{u}_{F}(k)$,

$$
\mathbf{u}_{F}(k)=\{\mathbf{u}(k \mid k), \mathbf{u}(k+1 \mid k), \cdots, \mathbf{u}(k+M-1 \mid k)\}
$$

Consider the sequence of $M-1$ inputs $\overline{\mathbf{u}}_{F}(k+1)$ given by

$$
\overline{\mathbf{u}}_{F}(k+1)=\{\mathbf{u}(k+1 \mid k), \cdots, \mathbf{u}(k+M-1 \mid k)\}
$$

then we have:

(i) The approximate reachable set contains all the predicted states for all possible realization of the uncertainties, that is

$$
\chi_{j}\left(\mathbf{x}_{k}, \mathbf{u}_{F}(k)\right) \subseteq \hat{X}_{j}\left(\mathbf{x}_{k}, \mathbf{u}_{F}(k)\right) \quad j=1, \cdots, M
$$

(ii) For any possible $\mathbf{x}_{k+1}=f\left(\mathbf{x}_{k}, \mathbf{u}(k \mid k)\right)+\mathbf{w}_{k}$, then

$$
\hat{X}_{j}\left(\mathbf{x}_{k+1}, \overline{\mathbf{u}}_{F}(k+1)\right) \subseteq \hat{X}_{j+1}\left(\mathbf{x}_{k}, \mathbf{u}_{F}(k)\right) \quad j=1, \cdots, M-1
$$

Proof: Both properties are proved by induction.

(i) From the definition we have that $\hat{X}_{1}\left(\mathbf{x}_{k}, \mathbf{u}_{F}(k)\right)=\chi_{1}\left(\mathbf{x}_{k}, \mathbf{u}_{F}(k)\right)$. Assume that $X_{j-1}\left(\mathbf{x}_{k}, \mathbf{u}_{F}(k)\right) \subseteq$ $\hat{X}_{j-1}\left(\mathbf{x}_{k}, \mathbf{u}_{F}(k)\right)$, then

$$
\begin{aligned}
X_{j}\left(\mathbf{x}_{k}, \mathbf{u}_{F}(k)\right) & =f\left(X_{j-1}, \mathbf{u}(k+j-1 \mid k)\right)+W \subseteq \psi\left(X_{j-1}, \mathbf{u}(k+j-1 \mid k)\right)+W \\
& \subseteq \psi\left(\hat{X}_{j-1}, \mathbf{u}(k+j-1 \mid k)\right)+W=\hat{X}_{j}\left(\mathbf{x}_{k}, \mathbf{u}_{F}(k)\right)
\end{aligned}
$$

(ii) It is clear that $\mathbf{x}_{k+1} \in \hat{X}_{1}\left(\mathbf{x}_{k}, \mathbf{u}_{F}(k)\right)$. In virtue of the monotony condition of $\psi(\cdot, \cdot)$, we have

$$
\begin{aligned}
\hat{X}_{1}\left(\mathbf{x}_{k+1}, \overline{\mathbf{u}}_{F}(k+1)\right) & =\psi\left(\mathbf{x}_{k+1}, \mathbf{u}(k+1 \mid k)\right)+W \\
& \subseteq \psi\left(\hat{X}_{1}\left(\mathbf{x}_{k}, \mathbf{u}_{F}(k)\right), \mathbf{u}(k+1 \mid k)\right)+W=\hat{X}_{2}\left(\mathbf{x}_{k}, \mathbf{u}_{F}(k)\right)
\end{aligned}
$$


Consider that $\hat{X}_{j-1}\left(\mathbf{x}_{k+1}, \overline{\mathbf{u}}_{F}(k+1)\right) \subseteq \hat{X}_{j}\left(\mathbf{x}_{k}, \mathbf{u}_{F}(k)\right)$. From the inclusion condition of $\psi(\cdot, \cdot)$ we have

$$
\begin{aligned}
\hat{X}_{j}\left(\mathbf{x}_{k+1}, \overline{\mathbf{u}}_{F}(k+1)\right) & =\psi\left(\hat{X}_{j-1}\left(\mathbf{x}_{k+1}, \overline{\mathbf{u}}_{F}(k+1)\right), \mathbf{u}(k+j \mid k)\right)+W \\
& \subseteq \psi\left(\hat{X}_{j}\left(\mathbf{x}_{k}, \mathbf{u}_{F}(k)\right), \mathbf{u}(k+j \mid k)+W=\hat{X}_{j+1}\left(\mathbf{x}_{k}, \mathbf{u}_{F}(k)\right)\right.
\end{aligned}
$$

The first property proves that assumption 1 suffices to compute approximate reachable sets; the second property establishes that the obtained sets are consistent, that is, the sequence of approximate reachable sets computed at the next sampling time for the remaining control sequence is included in the sequence of approximate reachable sets computed at the current sampling time.

In order to implement the computation of the approximate reachable sets, it is necessary to find procedures that satisfy assumption 1. A procedure based on interval arithmetic is used in this paper. In the following section, some well-known results are shown.

\subsection{Interval arithmetic}

Interval mathematics is a generalization of real mathematics in which interval numbers replace real numbers, interval arithmetic replaces real arithmetic, and interval analysis replaces real analysis [11]. Interval arithmetic has been applied in numerical analysis and in the study of the solutions of equations in compact domains [12], bounding the solution of ordinary differential equations [13] and global optimization problems $[14,15,16]$.

An interval number $X=[a, b]$ is the set of real numbers such that $\{x: a \leq x \leq b\}$. The same concept is extended to interval vectors, where each component is an interval variable. Note that an interval vector $X$ is a set in $\mathbb{R}^{n}$. The set of real compact intervals $[a, b], a, b \in \mathbb{R}$ is denoted by $I$, and the sets of interval vectors in $\mathbb{R}^{n}$ is denoted by $I^{n}$.

Interval arithmetic is an arithmetic defined on sets of intervals, instead of sets of real numbers. The four basic interval operations [11] are given by

$$
\begin{aligned}
{[a, b]+[c, d] } & =[a+c, b+d] \\
{[a, b]-[c, d] } & =[a-d, b-c] \\
{[a, b] \times[c, d] } & =[\min (a \cdot c, a \cdot d, b \cdot c, b \cdot d), \max (a \cdot c, a \cdot d, b \cdot c, b \cdot d)] \\
{[a, b] /[c, d] } & =[a, b] \times\left[\frac{1}{d}, \frac{1}{c}\right] \text { if } 0 \notin[c, d]
\end{aligned}
$$


The ranges of the four elementary interval arithmetic operations are exactly the ranges of the corresponding real operations. Extension of the interval arithmetic to include 0 in division can be found in [17]. The interval extension of standard functions, such as sin, cos, tan, arctan, exp, ln, abs, sqrt, is also possible.

Consider a function $g: \mathbb{R}^{n} \rightarrow \mathbb{R}^{m}$ and consider an interval vector $X \in I^{n}$, then the set $g(X)$ denotes the range of $g(\cdot)$ over the interval $X$. Note that it is not an interval vector in general. Computing the exact range of an arbitrary function $g(\cdot)$ over an interval vector $X$ is a difficult problem. However, interval arithmetic can be used to obtain interval bounds of the exact range $g(X)$.

Definition 3 (Inclusion function) A function $G: \mathbb{R}^{n} \rightarrow \mathbb{R}^{m}$ is called an inclusion function for $g(\cdot)$ if $g(X) \subseteq G(X)$ for any $X$ of $I^{n}$.

Definition 4 (Inclusion monotonic function) The inclusion function $G(\cdot)$ is inclusion monotonic if for every $X, Y \in I$ such that $X \subseteq Y$ it is satisfied that $G(X) \subseteq G(Y)$.

Definition 5 (Natural interval extension [14]) If $g: \mathbb{R}^{n} \rightarrow \mathbb{R}^{m}$ is a function computable as an expression, algorithm or computer program involving the four elementary operations interspersed with evaluations of standard functions, then a natural interval extension of $g(\cdot)$ is obtained by replacing each occurrence of each component $x_{i}$ of $\mathbf{x}$ by the corresponding interval $X_{i}$ of $X$, by executing all operations according to the formulas (8) and by computing exact ranges of standard functions.

Note that a natural interval extension of a function $g(\mathbf{x}): \mathbb{R}^{n} \rightarrow \mathbb{R}^{m}$ is a function $\psi(X): I^{n} \rightarrow I^{m}$.

Theorem 1 ([14]) Natural interval extensions are inclusion monotonic functions, i.e. for any $X \in I^{n}$, $g(X) \subseteq \psi(X)$ and for any $X \subseteq Y, \psi(X) \subseteq \psi(Y)$.

The conclusion is that natural interval extensions can be obtained for any function or any procedure. Bounds on the ranges can be computed from any expansion (rational, Taylor series, etc.) that has an explicit formula for the error term.

Now, let $\psi(X, \mathbf{u})$ be a natural interval extension of the model $f(\mathbf{x}, \mathbf{u})$, considering the inputs $\mathbf{u}$ as a parameter. Hence, it can be used as inclusion functions. From theorem 1, we get that for any $X, Y \in I^{n}$ such that $X \subseteq Y$ and for any $\mathbf{u}, f(X, \mathbf{u}) \subseteq \psi(X, \mathbf{u})$, and $\psi(X, \mathbf{u}) \subseteq \psi(Y, \mathbf{u})$.

Therefore this procedure satisfies assumption 1 and it can be used to compute the approximate reachable sets. Note that the set of uncertainties $W$ must be an interval vector, since the set $\psi(X, \mathbf{u})+W$ must be an 
interval vector in order to compute the following set in (5). This is a mild condition since an interval vector which contains $W$ can be used for the computation of the approximate reachable sets.

It is worth noting that the computational cost of the evaluation of the procedure $\psi(X, \mathbf{u})$ is of the same order of complexity that the evaluation of the function $f(x, \mathbf{u})$ (see for instance the interval extensions of the basic arithmetic operations, where an interval product operation requires at most 8 scalar products and 6 comparisons).

The approximate character of the obtained interval ranges can be reduced using several methods.

- Analyzing the function, reordering and grouping terms to reduce the so-called multi-incidence problem. This problem appears when a variable is repeated in an expression [14]. For example, when interval arithmetic evaluates an expression like $\mathrm{x}-\mathrm{x}$, the result is an outer approximation of the real solution.

- If the model function does not satisfy some monotony condition [15, remark 3.2], then the range of the function can not be exactly enclosed by an interval vector. In this case, when the sequence of approximate reachable sets is computed, the so-called wrapping effect may appear. This problem has been widely studied and some procedures to overcome it have been proposed [11, 12, 15]. In [13], the intervals have been extended to the notion of zonotopes: a zonotope is an affine mapping of an hypercube and it is quite more general than standard intervals. In this case, zonotopes can be used to obtain tighter approximations of the range of a function. The Kühn's method has been used in [18] to obtain tighter approximations of the reachable sets.

- Using a pre-stabilization structure: in this case, the control action $u_{k}$ is given by $u_{k}=K\left(x_{k}\right)+v_{k}$, where $K\left(x_{k}\right)$ is a given controller and $v_{k}$ is the new control input; thus the system is given by

$$
x_{k+1}=f\left(x_{k}, u_{k}\right)=f\left(x_{k}, K\left(x_{k}\right)+v_{k}\right)=f_{K}\left(x_{k}, v_{k}\right)
$$

The control law $K(x)$ is designed to stabilize the system or merely to reduce the error of the approximation of the interval extension. That is, it is designed to obtain a function $f_{K}(x, v)$ such that its interval extension provides better approximations. It can be obtained for instance to cancel terms which induce large errors in the interval extension.

Once we have a method to compute the approximate reachable sets, these are used to design a new 
robust MPC controller. This technique is presented in the following section.

\section{Robust MPC strategy}

Model predictive control is a well established optimal control strategy which considers constraints on the states and on the control actions [1]. The control law $K_{M P C}\left(\mathbf{x}_{k}\right)$ is obtained solving a constrained optimization problem and applying the optimal control action to the system in a receding horizon manner. Consider the finite horizon MPC optimization problem stated as follows

$$
\min _{\mathbf{u}_{F}(k)} J_{N}\left(\mathbf{x}_{k}, \mathbf{u}_{F}(k)\right)=\sum_{i=0}^{N-1} L(\hat{\mathbf{x}}(k+i \mid k), \mathbf{u}(k+i \mid k))+V(\hat{\mathbf{x}}(k+N \mid k))
$$

subject to:

$$
\begin{array}{ll}
\hat{\mathbf{x}}(k+j \mid k) \in X & \forall j=1, \cdots, N \\
\mathbf{u}(k+j \mid k) \in U & \forall j=0, \cdots, N-1 \\
\hat{\mathbf{x}}(k+N \mid k) \in \Omega &
\end{array}
$$

where the vector of decision variables $\mathbf{u}_{F}(k)=\{\mathbf{u}(k \mid k), \mathbf{u}(k+1 \mid k), \cdots, \mathbf{u}(k+N-1 \mid k)\}$ denotes the future sequence of control inputs of the system along the prediction horizon $N$ and $\hat{\mathbf{x}}(k+i \mid k)$ is the predicted nominal state of the system applying $\mathbf{u}_{F}(k) . L(x, u)$ is the so-called stage cost, which is a semi-definite positive function. Notice that the MPC includes a terminal $\operatorname{cost} V(\cdot)$ in the cost function and a terminal constraint given by the region $\Omega$.

Taking into account that the optimal minimizer $\mathbf{u}_{F}^{*}\left(\mathbf{x}_{k}\right)$ only depends on $\mathbf{x}_{k}$, and the receding horizon policy, the control law is given by $\mathbf{u}_{k}=K_{M P C}\left(\mathbf{x}_{k}\right)=\mathbf{u}^{*}(k \mid k)$. In absence of uncertainties, this control law asymptotically stabilizes the system under some assumptions on the terminal cost and the terminal region [1]. Moreover, the optimal cost function $J_{N}^{*}\left(\mathbf{x}_{k}\right)$ is a Lyapunov function of the closed loop system. The domain of attraction of the controller $X_{N}$ is the set where the optimization problem is feasible.

If the system is uncertain, then stability, and probably, feasibility of the nominal MPC may be lost. In [8] a terminal constraint is added to the MPC and dual-mode controller is proposed. The terminal set is considered a subset of a robust invariant set to ensure robust stability. Based on the Lipschitz continuity of the model, a bound of the uncertainties such that the uncertain system is stabilized is given. Due to the global nature of the Lipschitz constant, the obtained bound may be over-conservative. 
In this paper a robust dual-mode MPC is proposed. It is based on the computation of the approximate reachable sets shown in section 2.2. These sets allow us to consider all possible realizations of the uncertainties in the computation of the MPC control law. It is worth pointing out that the approximate reachable sets are local bounds on the effect of the uncertainties and hence, less conservative than global bounds based on a global Lipschitz constant.

In what follows, it is considered that the system satisfies the following assumption.

Assumption 2 There is a region $\Omega \subseteq X$ with an associated control law $\mathbf{u}=h(\mathbf{x})$ such that $\Omega$ is an admissible robust positively invariant set for the uncertain system. That is, if $\mathbf{x} \in \Omega$, then $h(\mathbf{x}) \in U$ and $f(\mathbf{x}, h(\mathbf{x}))+\mathbf{w} \in \Omega, \forall \mathbf{w} \in W$.

There exists well-established methods to compute robust controllers for nonlinear systems [19]. If an associated robust Lyapunov function is obtained then it can be used as terminal cost and a level set can be used as terminal set. This choice can be considered as a quasi-infinite prediction horizon, and hence it provides an enhanced closed loop performance [20].

Note that we only require that this controller robustly stabilizes the system in a neighborhood of the steady state; this allows us to use local approximation to the nonlinear system around the steady state. Thus, a linear approximation can be used in a similar way that the proposed one in [20]. Another technique is approximating the nonlinear system by a linear differential inclusion (LDI) and compute a robust linear controller and the maximal robust invariant set, which is a polyhedron [21].

The proposed controller is derived from the following optimization problem:

Robust dual-mode MPC optimization problem $\left(P_{k}^{d}\left(\mathbf{x}_{k}\right)\right)$

$$
\begin{aligned}
\min _{\mathbf{u}_{F}(k)} & J_{N-k}\left(\mathbf{x}_{k}, \mathbf{u}_{F}(k)\right)=\sum_{i=0}^{N-k-1} L(\hat{\mathbf{x}}(k+i \mid k), \mathbf{u}(k+i \mid k))+V(\hat{\mathbf{x}}(N \mid k)) \\
\text { s.t } & \hat{X}_{j}\left(\mathbf{x}_{k}, \mathbf{u}_{F}(k)\right) \subseteq X \quad \forall j=1, \cdots, N-k \\
& \mathbf{u}(k+j \mid k) \in U \quad \forall j=0, \cdots, N-k-1 \\
& \hat{X}_{N-k}\left(\mathbf{x}_{k}, \mathbf{u}_{F}(k)\right) \subseteq \Omega
\end{aligned}
$$

The robust dual-mode control law is such that when the system is not in the terminal region, then the solution of $P_{k}^{d}\left(\mathbf{x}_{k}\right)$ is applied, and when the system is in the terminal region, then the local robust control 
law is applied. Thus, the dual-mode control law is given by

$$
K_{M P C}^{d}\left(\mathbf{x}_{k}\right)= \begin{cases}\mathbf{u}^{*}(k \mid k) & \text { if } \mathbf{x}_{k} \notin \Omega \\ h\left(\mathbf{x}_{k}\right) & \text { if } \mathbf{x}_{k} \in \Omega\end{cases}
$$

where $\mathbf{u}^{*}(k \mid k)$ is the first control input of $\mathbf{u}_{F}^{*}(k)$, solution to the optimization problem $P_{k}^{d}\left(\mathbf{x}_{k}\right)$.

Note that the control horizon of the optimization problem is reduced at each sample time. Therefore, this optimization problem is only defined for $k=0$ to $k=N-1$. In the next section it will be proved that the system reaches the terminal region in $N$ steps, i.e. $\mathbf{x}_{N} \in \Omega$, and, hence, the local control law $\mathbf{u}=h(\mathbf{x})$ makes the system remains in $\Omega$. Consequently, the controller is well defined.

The approach proposed in this paper is different to the one proposed in [8]: the notion of reachable set is added and hence the effect of the uncertainty is considered along the control horizon. This fact allows us to consider the constraints on the states in a more natural way. Consequently, it is not necessary to use a more conservative terminal region as in [8].

We propose the use of local procedures for the computation of the approximate reachable sets. This constitutes the main difference with respect to [8], where a global Lipschitz constant is used. Hence, our method is potentially less conservative, which leads to a larger domain of attraction.

\section{Stability analysis}

Since the uncertainties are merely bounded and they may not be decaying, the origin is not a steady state of the uncertain system. Hence, the aim of a stabilizing controller is to steer the state to a neighborhood of the origin and keep the state evolution in it. This set is a robust positively invariant set for the closed loop system and its size depends on the bound on the uncertainties. Therefore, the notion of asymptotic stability is not suitable and the definition of system ultimately bounded is introduced:

Definition 6 ([19]) A system is asymptotically ultimately bounded if the system evolves asymptotically to a bounded set, i.e. there exist positive constants $b$ and $c$ such that for every $\alpha \in(0, c)$, there is a $k^{*}$ such that for all $\left\|\mathbf{x}_{0}\right\| \leq \alpha$ then $\left\|\mathbf{x}_{k}\right\| \leq b, \forall k>k^{*}$.

This definition of stability is closely related to the notion of Input-to-state stability (ISS). In this case, sufficient stability conditions are imposed by means of the so-called ISS-Lyapunov function. See for instance [22] where an ISS MPC controller is proposed. 
As it is proved in the following theorem, for any feasible initial state, the proposed controller steers the uncertain system to the terminal region where it remains for all the time. Hence, the closed loop system is ultimately bounded.

Theorem 2 (Stability) Consider a system (1) with additive uncertainties subject to (2) and with constraints on the states $\mathbf{x}_{k} \in X$ and on the inputs $\mathbf{u}_{k} \in U$. Consider a robust invariant set for the system $\Omega$ with an associated local controller $\mathbf{u}=h(\mathbf{x})$ such that both satisfy assumption 2. Consider that a procedure to compute the approximate reachable sets satisfying assumption 1 is available. Then the system controlled by $\mathbf{u}=K_{M P C}^{d}\left(\mathbf{x}_{k}\right)$ is ultimately bounded for all $\mathbf{x}_{0}$ such that the optimization problem $P_{0}^{d}\left(\mathbf{x}_{0}\right)$ is feasible.

Proof: The stability is based on the feasibility of the optimization problem for all the time. That is, if the initial state $x_{0}$ is feasible, then the optimization problem $P_{k}^{d}\left(x_{k}\right)$ is feasible for all $k \geq 0$. Since the control horizon is reduced at each sampling time, the system reaches the terminal region in $N$ steps. Once the system is in $\Omega$, the controller switches to $h(x)$ and this controller makes the system never leave $\Omega$.

Feasibility is proved by induction. By assumption, $\mathbf{x}_{0}$ is such that the optimization problem $P_{o}^{d}\left(\mathbf{x}_{0}\right)$ is feasible. Assume that in $\mathbf{x}_{k-1}$ the optimization problem $P_{k-1}^{d}\left(\mathbf{x}_{k-1}\right)$ is feasible and the optimal (a feasible) solution is

$$
\mathbf{u}_{F}^{*}(k-1)=\left\{\mathbf{u}^{*}(k-1 \mid k-1), \mathbf{u}^{*}(k \mid k-1), \cdots, \mathbf{u}^{*}(N-1 \mid k-1)\right\}
$$

Let $\mathbf{x}_{k}=f\left(x_{k-1}, K_{M P C}^{d}\left(x_{k-1}\right)\right)+w_{k-1}$ be the state where the uncertain system evolves at $k$, and let $\overline{\mathbf{u}}_{F}(k)$ be a sequence of control inputs given by

$$
\overline{\mathbf{u}}_{F}(k)=\left\{\mathbf{u}^{*}(k \mid k-1), \mathbf{u}^{*}(k+1 \mid k-1), \cdots, \mathbf{u}^{*}(N-1 \mid k-1)\right\}
$$

then we are going to prove that $\overline{\mathbf{u}}_{F}(k)$ is a feasible solution to $P_{k}^{d}\left(x_{k}\right)$ for all possible uncertainty $w_{k-1} \in W$.

- Input constraints: Since $\mathbf{u}_{F}^{*}(k-1)$ is a feasible solution to $P_{k-1}^{d}\left(x_{k-1}\right)$, then $u^{*}(j \mid k-1) \in U$ for all $j=k-1, \cdots, N-1$. Therefore, from (12) we derive that $\overline{\mathbf{u}}_{F}(k)$ is admissible.

- State constraints: It is clear that $\mathbf{x}_{k} \in \hat{X}_{1}\left(\mathbf{x}_{k-1}, \mathbf{u}_{F}^{*}(k-1)\right)$; then in virtue of property 1 we have that

$$
\hat{X}_{j-1}\left(\mathbf{x}_{k}, \overline{\mathbf{u}}_{F}(k)\right) \subseteq \hat{X}_{j}\left(\mathbf{x}_{k-1}, \mathbf{u}_{F}^{*}(k-1)\right) \subseteq X \quad \text { for } \quad j=2, \cdots, N-k+1
$$

- Terminal constraint: From the state constraints we also derive that

$$
\hat{X}_{N-k}\left(\mathbf{x}_{k}, \overline{\mathbf{u}}_{F}(k)\right) \subseteq \hat{X}_{N-k+1}\left(\mathbf{x}_{k-1}, \mathbf{u}_{F}^{*}(k-1)\right) \subseteq \Omega
$$


Therefore, $\overline{\mathbf{u}}_{F}(k)$ is a feasible solution of $P_{k}^{d}\left(\mathbf{x}_{k}\right)$ and by induction, the optimization problem is feasible for all the time.

Now, we are going to prove that the state of the closed loop system at time $N$ is in the terminal region, i.e. $\mathbf{x}_{N} \in \Omega$. In effect, from (13), we have that at sampling time $k=N-1$, the control action must guarantee that $\hat{X}_{1}\left(\mathbf{x}_{N-1}, \mathbf{u}^{*}(N-1 \mid N-1)\right) \subseteq \Omega$, and hence, due to $x_{N} \in \hat{X}_{1}\left(\mathbf{x}_{N-1}, \mathbf{u}^{*}(N-1 \mid N-1)\right)$ for all $w_{N-1} \in W$, we derive that $x_{N} \in \Omega$.

Once the system reaches the terminal set the controller switches to the local controller $\mathbf{u}=h(\mathbf{x})$ which, by assumption 2 , guarantees that the closed loop system evolution remains into the terminal region $\Omega$. Thus, the closed loop system is ultimately bounded.

Remark 1 Note that the stability is guaranteed thanks to the feasibility of the computed control action at each sample time. Hence, optimality is not required and a suboptimal solution of the optimization problem suffices to guarantee stability. This property allows us to relax the computational burden of the optimization problem.

Moreover, from the stability proof we derive that at each sampling time we can compute an initial feasible solution based on the solution obtained at the previous sampling time; this initial state is a hot start for the optimization problem. This property and the relaxation of the optimality requirement allow us to reduce the computational burden necessary to compute the control action at each sampling time.

Remark 2 The feasibility is guaranteed by means of the reduction of the control horizon at each sampling time. In order to maintain the horizon considered in the cost to minimize, a constant prediction horizon $N_{p}$ can be considered. In this case, the local control law is used to predict the evolution from the control horizon to the prediction horizon [23]. This is equivalent to use he following modified terminal cost

$$
V(\hat{x}(N \mid k))=\sum_{j=N-k}^{N_{p}} L(\hat{x}(k+j \mid k), h(\hat{x}(k+j \mid k))
$$

where $\hat{x}(k+j+1 \mid k)=f(\hat{x}(k+j \mid k), h(\hat{x}(k+j \mid k)))$ for $j=N-k, \cdots, N_{p}$.

Note that stability is independent on both the stage cost and the terminal cost. Thus, this choice of the terminal cost function has only effect on the performance but not on the stability of the closed-loop system. The proposed controller can be extended to fixed control horizon considering the robust invariance condition [24]. 
Remark 3 The proposed controller is able to stabilize the system at any feasible initial state. Thus, the size of the domain of attraction of the closed loop system is related with the considered bounds of the uncertainties; in fact, if the uncertainty is reduced, the domain of attraction is enlarged. The open-loop nature of the proposed robust MPC makes the controller conservative in the sense that the resulting domain of attraction is probably smaller than the robustly stabilizable region.

This conservativeness can be reduced considering a pre-stabilization policy. This provides some degree of feedback in the prediction (although it is not a closed-loop formulation). Note that in this case, the input constraints are interval constraints, since an interval estimation of the control action for every approximate reachable set is required.

Additionally, this technique can also improve the accuracy of the approximation of the reachable sets, as it was shown in the section 2.3. Another technique to reduce the conservativeness is to consider zonotopic estimations of the approximate reachable sets [18].

\section{Application to a CSTR model}

To illustrate the proposed robust MPC controller, it is applied to a benchmark system [23]: the continuous stirred tank reactor (CSTR). A CSTR for an exothermic, irreversible reaction $A \rightarrow B$ with constant liquid volume is considered. The continuous time model is derived from the mass and energy balances and it is given by $[25,23]$ :

$$
\begin{aligned}
\frac{d C_{A}}{d t} & =\frac{q}{V} \cdot\left(C_{A f}-C_{A}\right)-k_{0} \cdot \exp \left(-\frac{E}{R \cdot T}\right) \cdot C_{A}+w_{C_{A}} \\
\frac{d T}{d t} & =\frac{q}{V} \cdot\left(T_{f}-T\right)-\frac{\Delta H \cdot k_{0}}{\rho \cdot C_{p}} \cdot \exp \left(-\frac{E}{R \cdot T}\right) \cdot C_{A}+\frac{U \cdot A}{V \cdot \rho \cdot C_{p}} \cdot\left(T_{c}-T\right)+w_{T}
\end{aligned}
$$

where $C_{A}$ is the concentration of $A$ in the reactor, $T$ is the reactor temperature and $T_{c}$ is the temperature of the coolant stream. $w_{C_{A}}$ and $w_{T}$ model the uncertainty on both states.

The considered parameters of the model [23]: $\rho=1000 \mathrm{~g} / \mathrm{l}, C_{p}=0.239 \mathrm{~J} / \mathrm{g} \mathrm{K}, \Delta H=-5 \times 10^{4} \mathrm{~J} / \mathrm{mol}$, $E / R=8750 \mathrm{~K}, k_{0}=7.2 \times 10^{10} \mathrm{~min}^{-1}, U \cdot A=5 \times 10^{4} \mathrm{~J} / \mathrm{min} \mathrm{K}$. The nominal operating conditions are given by[23]: $q=100 \mathrm{l} / \mathrm{min}, T_{f}=350 \mathrm{~K}, V=1001, C_{A f}=1.0 \mathrm{~mol} / \mathrm{l}$. In these conditions, the steady state is $C_{A}^{o}=0.5 \mathrm{~mol} / \mathrm{l}, T^{o}=350 \mathrm{~K}$ and $T_{c}^{o}=300 \mathrm{~K}$, which is an unstable equilibrium point. The temperature of the coolant is constrained to $280 \mathrm{~K} \leq T_{c} \leq 370 \mathrm{~K}$, the concentration of $A$ is constrained to $0.4 \mathrm{~mol} / 1 \leq C_{A} \leq$ 
$0.6 \mathrm{~mol} / 1$. As in [23], the model is discretized using a sampling period $T_{s}=0.03 \mathrm{~min}$. We consider that the uncertainties are bounded by

$$
\left|w_{C_{A}}\right| \leq 0.1 \mathrm{~mol} /(1 \mathrm{~min}) \text { and }\left|w_{T}\right| \leq 8^{\circ} \mathrm{K} / \mathrm{min}
$$

The objective is to regulate in an admissible way the concentration $C_{A}$ and the reactor temperature $T$ around the steady state manipulating the temperature of the coolant in its admissible range, for any possible uncertainty.

In order to improve the robust controller, a pre-stabilization structure is considered. This controller stabilizes locally the system, thus it is used also as local control law for the dual-mode MPC controller. The control law is

$$
K(x)=\left(33.46-7.2 \cdot 10^{12} \exp \left(-\frac{E}{R \cdot T}\right)\right) \cdot C_{A}-1.868 \cdot T+987.07
$$

The closed loop system has been approximated by a LDI to compute a robust invariant set $\Omega$. The obtained polytope is used as terminal region in the MPC formulation. As terminal cost is considered a cost function given by (14), with $N p=50$. The considered stage cost $L(\mathbf{x}, u)=\mathbf{x}^{T} \cdot \mathbf{Q} \cdot \mathbf{x}+u^{T} \cdot R \cdot u$, with

$$
\mathbf{Q}=\left[\begin{array}{cc}
2.0 & 0 \\
0 & 2.9 \cdot 10^{-3}
\end{array}\right] \text { and } R=3.33 \cdot 10^{-3}
$$

as in [23]. The MPC controller has been executed with a control horizon $N=15$. In figure 1 , the sequence of $N$ approximate reachable sets computed for the optimal solution in a given initial state is shown.

To illustrate the evolution of the system, the uncertainty has been considered as constant along the time with an extreme value. Thus, four different scenarios have been considered: scenario \#1 with $w_{C_{A}}=0.1$ and $w_{T}=8$, scenario \#2 with $w_{C_{A}}=0.1$ and $w_{T}=-8$, scenario \#3 with $w_{C_{A}}=-0.1$ and $w_{T}=-8$ and scenario \#4 with $w_{C_{A}}=-0.1$ and $w_{T}=8$. The state portrait of the closed loop evolution for several initial points in the four considered scenarios are depicted in figures 2, 3, 4 and 5 respectively. In these ones, the admissible convergence of the closed loop system in spite of the uncertainties is demonstrated. Moreover, the collection of the chosen initial state shows the size of the domain of attraction of the controller. It is worth remarking that in these figures, the system evolves to an steady state which is different at each scenario due to the uncertainties; the steady state is depicted with a circle. Note how the uncertainties affect to this steady state, which gives an idea of the amount of the uncertainty considered. 


\section{Conclusions}

In this paper, a robust dual-mode MPC controller for constrained discrete-time nonlinear systems with additive uncertainties is presented. It is based on the addition of the uncertain prediction of the system in the MPC optimization problem. This is done via the so-called approximate reachable sets, which provide a tractable way of considering the effect of the uncertainties on the predictions. It has been demonstrated that interval arithmetic is an appropriate and tractable technique for the on-line computation of the approximate reachable sets.

Based on the computation of the approximate reachable sets, a robust dual-mode MPC strategy is proposed. Considering a robust positively invariant set as terminal region, any feasible initial state is robustly steered to the terminal set, where it remains. Thus, under feasibility of the optimization problem in the initial state, robust stability and feasibility of the closed-loop system is guaranteed. The local character of the approximate reachable sets makes that the proposed controller improves previous robust dual-mode MPC formulations. Furthermore, suboptimal solution of the optimization problem guarantees stability and hence optimality is not necessary. Finally, the proposed controller has been applied to a CSTR model in order to illustrate some of its properties and how interval arithmetic is used to compute the uncertain evolution sets.

\section{Acknowledgements}

The authors would like to thank the anonymous reviewers and the editor for their helpful comments. We would like acknowledge also MCYT-Spain (contracts DPI-2001-2380-03-01 and DPI-2002-4375-C02-01) for funding this work.

\section{References}

[1] D. Q. Mayne, J. B. Rawlings, C. V. Rao, and P. O. M. Scokaert. Constrained model predictive control: Stability and optimality. Automatica, 36:789-814, 2000.

[2] E. F. Camacho and C. Bordons. Model Predictive Control. Springer-Verlag, 2 edition, 1999.

[3] S. J. Qin and T. A. Badgwell. A survey of industrial model predictive control technology. Control Engineering Practice, 11:733-764, 2003. 
[4] F. Allgöwer, T. A. Badgwell, J. S. Qin, J. B. Rawlings, and S. J. Wright. Nonlinear model predictive control and moving horizon estimation - an introductory overview. In P. M. Frank, editor, Advances in Control, pages 391-449. Springer, 1999.

[5] D. Q. Mayne. Nonlinear model predictive control: Challenges and opportunities. In F.Allgöwer and A.Zheng, editors, Nonlinear Model Predictive Control, pages 23-44. Birkhauser, 2000.

[6] P. O. M. Scokaert, J. B. Rawlings, and E. S. Meadows. Discrete-time stability with perturbations: Application to model predictive control. Automatica, 33(3):463-470, 1997.

[7] D. Limon, T. Alamo, and E. F. Camacho. Stability analysis of systems with bounded additive uncertainties based on invariant sets: Stability and feasibility of MPC. In Proceedings of the ACC, 2002.

[8] H. Michalska and D. Q. Mayne. Robust receding horizon control of constrained nonlinear systems. IEEE Transactions on Automatic Control, 38(11):1623-1633, 1993.

[9] P. O. M. Scokaert and D. Q. Mayne. Min-max feedback model predictive control for constrained linear systems. IEEE Transactions on Automatic Control, 43(8):1136-1142, 1998.

[10] V. Sakizlis, N. M. P. Kakalis, V. Dua, J. D. Perkins, and E. N. Pistikopoulos. Design of robust modelbased controllers via parametric programming. Journal of Process Control, 40:189-201, 2004.

[11] E. Moore. Interval analysis. Prentice Hall, 1996.

[12] A. Neumaier. Interval methods for systems of equations. Cambridge University Press, 1990.

[13] W. Kühn. Rigorous computed orbits of dynamical systems without the wrapping effect. Computing, 61:47-67, 1998.

[14] R. B. Keartfort. Rigorous global search: continuous problems. Kluwer Academic Publishers, 1996.

[15] J. Papamichail and C. S. Adjiman. A rigorous global optimization algorithm for problems with ordinary differential equations. Journal of Global Optimization, 24:1-33, 2002.

[16] W. R. Esposito and C. A. Floudas. Global optimization for the parameter estimation of differentialalgebraic systems. Ind. Eng. Chem. Res, 39:1291-1310, 2000.

[17] E. Hansen. Global optimization using interval analysis. Marcel Dekker, Inc., 1992. 
[18] J. M. Bravo, D. Limon, T. Alamo, and E.F. Camacho. Robust MPC of constrained discrete-time nonlinear systems based on zonotopes. In European Control Conference, 2003.

[19] H. Khalil. Nonlinear Systems. Prentice-Hall, 2 edition, 1996.

[20] H. Chen and F. Allgöwer. A quasi-infinite horizon nonlinear model predictive control scheme with guaranteed stability. Automatica, 34(10):1205-1218, 1998.

[21] F. Blanchini. Set invariance in control. Automatica, 35:1747-1767, 1999.

[22] D. Limon, T. Alamo, and E. F. Camacho. Input-to-state stable MPC for constrained discrete-time nonlinear systems with bounded additive uncertainties. In Proceedings of the CDC, 2002.

[23] L. Magni, G. De Nicolao, L. Magnani, and R. Scattolini. A stabilizing model-based predictive control algorithm for nonlinear systems. Automatica, 37:1351-1362, 2001.

[24] D. Limon. Control Predictivo de Sistemas no lineales con restricciones: estabilidad y robustez. PhD thesis, Universidad de Sevilla, 2002.

[25] D. E. Seborg, T.F. Edgar, and D.A. Mellichamp. Process Dynamics and Control. Wiley, 1989. 


\section{List of Figures}

1 Sequence of predicted approximate reachable sets of the optimal solution at $k=0 . \quad \ldots \quad$. 21

2 Scenario \#1: trajectories of the closed loop system. . . . . . . . . . . . . . 21

3 Scenario \#2: trajectories of the closed loop system. . . . . . . . . . . . . . . . . 21

4 Scenario \#3: trajectories of the closed loop system. . . . . . . . . . . . . . . . 22

5 Scenario \#4: trajectories of the closed loop system. . . . . . . . . . . . . . . 22 


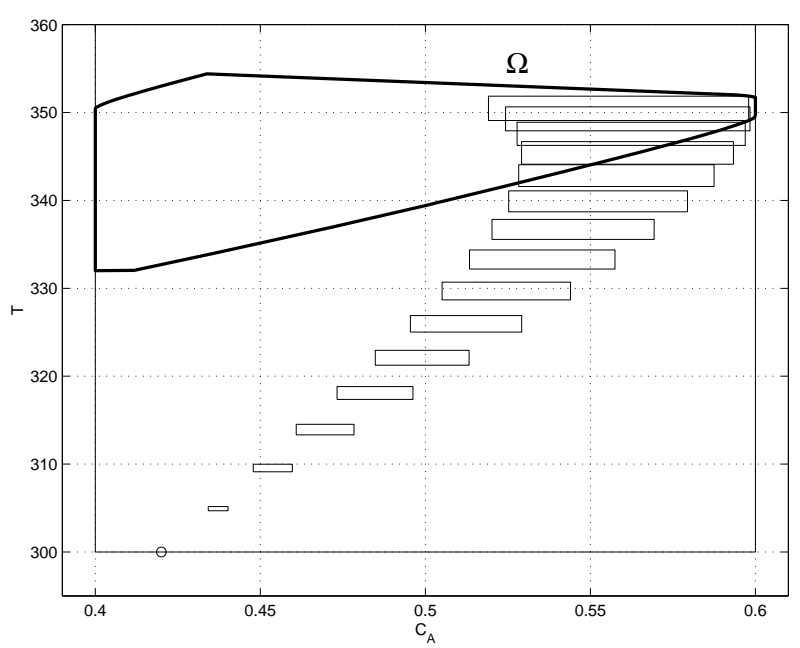

Figure 1: Sequence of predicted approximate reachable sets of the optimal solution at $k=0$.

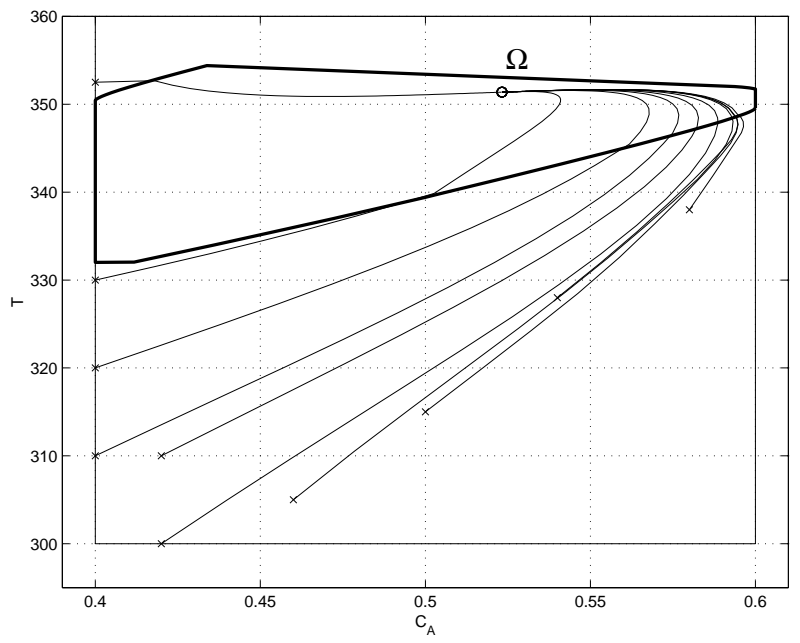

Figure 2: Scenario \#1: trajectories of the closed loop system.

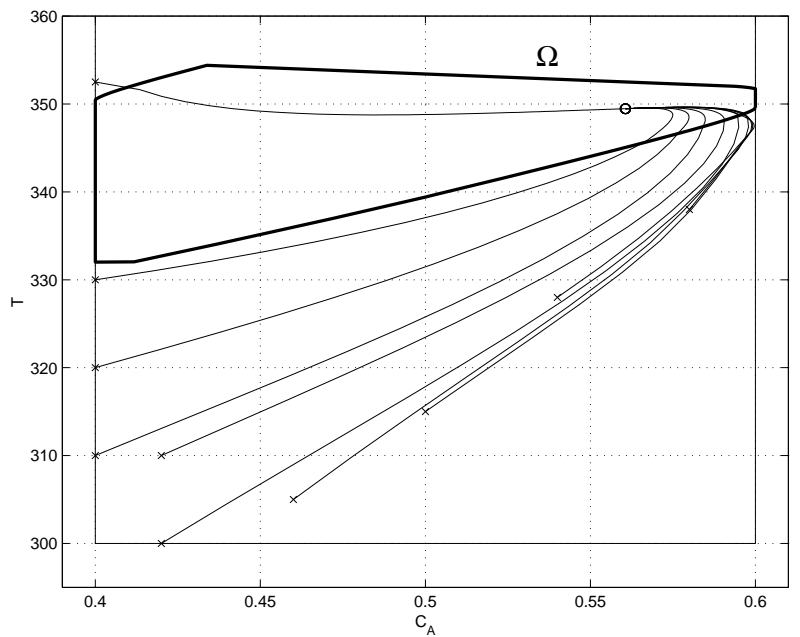

Figure 3: Scenario \#2: trajectories of the closed loop system. 


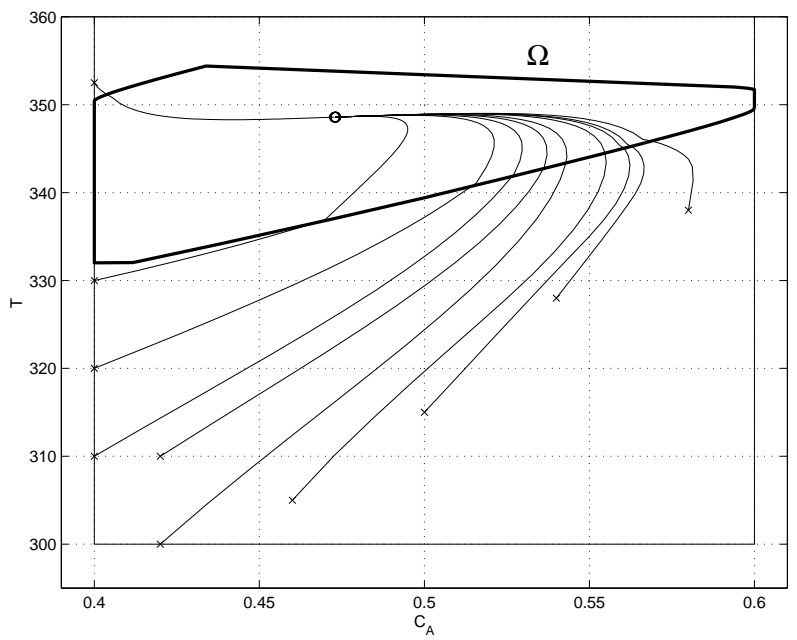

Figure 4: Scenario \#3: trajectories of the closed loop system.

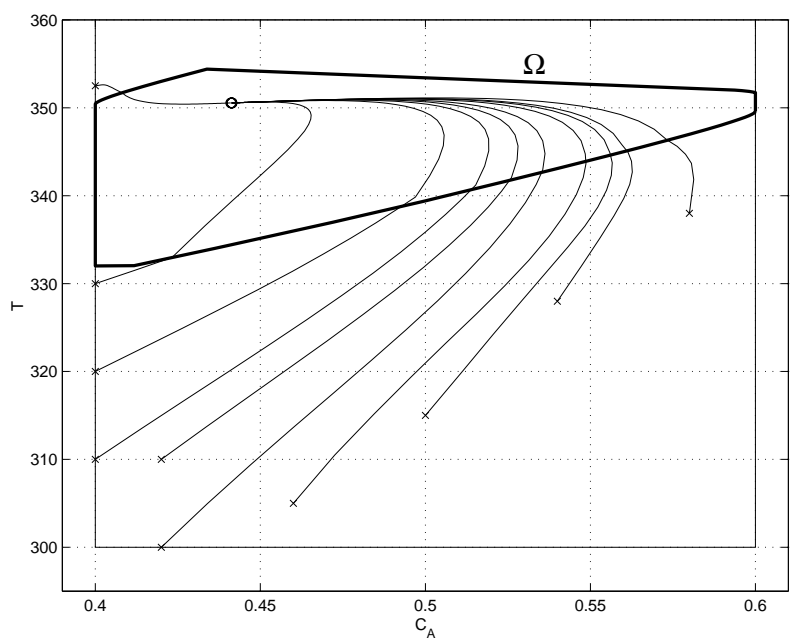

Figure 5: Scenario \#4: trajectories of the closed loop system. 